\title{
PREDICTION OF MONTHLY BODY WEIGHT FROM BODY MEASUREMENTS IN BEETAL GOATS REARED UNDER FIELD AND FARM CONDITIONS
}

\author{
H. M. Waheed ${ }^{1,2,3}$, M. Moaeen-ud-Din ${ }^{* 1,2}$, M. S. Khan ${ }^{2,3}$, M. Saif-ur-Rehman ${ }^{2,3}$ and M. S. Nawaz-ul-Rehman ${ }^{4}$ \\ ${ }^{1}$ Department of Animal Breeding and Genetics, Faculty of Veterinary and Animal Sciences, PMAS Arid Agriculture \\ University, Rawalpindi, 46300, Pakistan; ${ }^{2}$ National Center for Livestock Breeding, Genetics and Genomics, PMAS Arid \\ Agriculture University, Rawalpindi, 46300, Pakistan ${ }^{3}$ Institute of Animal and Dairy Sciences, Faculty of Animal \\ Husbandry, University of Agriculture, Faisalabad, 38000, Pakistan \\ ${ }^{4}$ Centre of Agricultural Biochemistry and Biotechnology (CABB), University of Agriculture, Faisalabad, 38000, \\ Pakistan \\ *Corresponding Author's Email: drmoinawan@gmail.com
}

https://doi.org/10.36899/JAPS.2020.1.0003

Published online January 02, 2020

\begin{abstract}
The aim of the present study was to predict live body weight from several body measurements in Beetal goats at various ages. Data on live body weight and linear body measurements taken from Beetal goats were recorded from six private herds and two government farms of Punjab, Pakistan. Final data file contained 5011 observations of live body weight (LBW) and different body measurements i.e. whither height (WH), body length (BL), chest girth (CG), chest width (CW) and pin bone width (PW). The quantitative traits were recorded by hanging balance (for LBW) and tailor's tape (for all others) from January 2016 to May 2017. The ages of animals were divided into thirty-seven classes with birth age class declared as 0 , monthly age class 1 (1-30 days) and so on up to monthly age class 36 (1051-1080d). The overall birth and 36 month averages of LBW, WH, BL, CG, CW and PW were found $3.34 \& 80.21(\mathrm{~kg}), 33.61 \& 92.91,25.11 \&$ $84.86,30.96 \& 93.30,8.88 \& 26.21$ and $4.69 \& 14.00(\mathrm{~cm})$, respectively. All the body measurements showed highly significant effect on LBW of animals at 3 and 21 months of age $(P \leq 0.05)$. The CG was highly significantly related to LBW in all monthly age classes with the exception of $0,1,2$ and 7 months $(\mathrm{P} \leq 0.05)$. The $\mathrm{PW}$ had non-significant effect on LWB for all monthly age classes with the exception of 3, 4, 9, 10,11, 21, 32 and 35 months. The WH had highly significant effect on LBW of animals in monthly age classes of 3, 7, 10,11,12, 14, 15, 16, 17, 20, 21 and 35. The BL in general showed significant effect on LBW up to 26 months of age. In agreement to regression results, CG had strong positive phenotypic correlation (0.921, $\mathrm{P} \leq 0.05)$ with $\mathrm{LBW}$, followed by $\mathrm{BL}(0.875, \mathrm{P} \leq 0.05)$, WH $(0.864, \mathrm{P} \leq 0.05), \mathrm{CW}$ $(0.777, \mathrm{P} \leq 0.05)$ and $\mathrm{PW}(0.725 \mathrm{P} \leq 0.05)$. The value of R-squared of linear regression model is 0.85 . In conclusion, $\mathrm{CG}$, $\mathrm{BL}, \mathrm{WH}, \mathrm{CW}$ and PW may be used for predicting live body weight in Beetal goat in the order of priority. These body measurements may be combined in an index with appropriate weights to more accurately predict live body weight under field conditions. Considering precisely estimated genetic parameters for all these traits, the body measurement traits may also be used for indirect genetic selection for improved growth rate in Beetal goat.
\end{abstract}

Key Words: Beetal goat, live body weight, body measurements, Pakistan.

\section{INTRODUCTION}

Goat population of the Pakistan is among the most rapidly increasing livestock species. Current population of goat is 76.1 million heads (GOP, 2018-19). Pakistan is not only $3^{\text {rd }}$ largest goat producing country in term of population of the world; but also, a large proportion of human population especially in the countryside have their livelihood associated with rearing of goat. Currently, 36 reported breeds of goat in Pakistan are available. Among these 36 goat breeds, Beetal is the largest in size followed by Kamori. It is considered the most important for producing large size animals, preferred on the occasion of Eid-ul-Azha (Khan et al., 2006; Khan et al., 2008). It is the second most common breed of Punjab after Teddy (GOP, 2006). Although, bigger size animals are possible in Beetal goat; however, more detailed information on growth rate and potential under farm and field conditions is still scarce.

Growth is a time dependent change in the weight or size of organ, composition of tissue/organ, size and number of cells as well as in live weight of an organism. It is a biological phenomenon that can be interpreted mathematically (Eisen, 1976; Waheed, 2011; Eder et al., 2017). Growth has important implications for domestic animal production as it significantly influences the value of the animal being produced (An et al., 2011). Growth, which is of great importance economically in goat production, can surely be considered as one of the most significant traits. Animal growth is continuous and dynamic process. 
Knowledge of body weight prediction in goats is essential for various reasons, i.e. the control and management of the flock during the whole rearing process, breeding (selection), feeding, health care (medication) and marketing of goats (Slippers et al., 2000; Pesmen and Yardimci, 2008). Farmers need to depend on inaccurate estimates of the body weights of their goat, leading to mistakes in decisions making (Khan et al., 2006). Predicting the live weight from body measurements is useful, faster, simpler and less expensive in the rural areas where the facilities are lacking for the breeders (Tsegaye et al., 2013). Animal live body weight is a critical element yet can rarely be measured in rural areas where reasonable precise scales are unavailable. In conclusion, as the body measurements has high association with live body weight, this may be used as selection criteria (Moela, 2014). It was reported that the relationship between body weight and linear body measurements in meat animals was examined in predicting body size and shape (Khan et al., 2006). Ultimately, when farmers and buyers of livestock are able to relate body measurement of animals to assess their LBW, optimum price and benefit will be given to owners.

Previous studies about the linear body measurements of the goats have been carried in various regions of the world within the scope of the prediction of their live body weight (Khan et al., 2006; Pesmen and Yardimci, 2008; Chitra et al., 2012; Tsegaye et al., 2013; Iqbal et al., 2013). In Pakistan, different studies regarding estimation of live-body weight through regression parameters of linear body measurements with Beetal and other goat breeds have been done with less number of records (Waheed, 2011; Iqbal et al., 2013; Eyduran et al., 2013; Eyduran et al., 2017). Live body-weight of the Beetal goats under field and farm condition with more number of linear body measurements has not yet been predicted. Therefore, an attempt was made in the present study to predict the live body-weight in Beetal goats reared under field or farm conditions using the regression analysis method.

\section{MATERIALS AND METHODS}

Growth performance data of the Beetal goats were recorded from field and farm area of Punjab Province. Different private herds of Beetal goats were visited and observations were recorded. While, two government farms i.e. Livestock Experiment Station (LES), Allahdad, tehsil Jahanian, district Khanewal, and Directorate of Farms, University of Agriculture, Faisalabad (UAF) were also visited and observations were taken. Ear tags and neckbands were used for identification of the handled animals. The age of government farm animals is determined from birth register while the herd owners of field animals provided the age record of their animals.
Live-weight and different body measurements were recorded between January 2016 and May 2017. A digital weighing scale (with a capacity of up to $500 \mathrm{~kg}$ ) was used at UAF to determine actual live weight, while at LES manual-weighing scale with same capacity was used. In field conditions (registered farms in Punjab) hanging balance with a capacity of $150 \mathrm{~kg}$ was used to determine actual live-weight. All types of weighing scales were checked and calibrated before each bodyweight record. After determining the live weight, the animal was made to stand upright on a flat ground area. All body measurements were taken with tailor measuring tape included wither height "WH", body length "BL", chest girth "CG", chest width "CW" and pin bone width "PW". The average time to take all these measurements was 15 seconds. All measurements were taken early in the morning before animals were fed and taken by the same person to avoid individual variation.

To predict the live body-weight of Beetal goats at any age, the observations were divided in classes on the basis of age. The ages of animals were divided into thirty-seven classes with birth age class declared as 0 , monthly age class 1 (1-30 days) and so on up to monthly age class 36 (1051-1080d). The animals above three year of age were also grouped in last class.

Microsoft Excel was used for entering and editing the data. General linear model and random regression model of SAS (SAS., 2017) were used to estimate the significance of different body measurements on live-weight of animals along with R-Square values for each age class and to estimate parameters of different body measurement for each age group along with RSquare values to predict live body weight of animals, respectively. Body weight was considered as response variable and $\mathrm{WH}, \mathrm{BL}, \mathrm{CG}, \mathrm{CW}$ and $\mathrm{PW}$ were taken as predictors. The regression model for each age class was:

$Y=\beta_{0}+\beta_{1} X_{1}+\beta_{2} X_{2}+\beta_{3} X_{3}+\beta_{4} X_{4}+\beta_{5} X_{5}$

Where,

$\mathrm{Y}=$ body weight

$\beta_{0}=$ intercept

$\beta_{1} \ldots \beta_{5}=$ regression coefficients

$\mathrm{X}_{1} \ldots \mathrm{X}_{5}=$ predictor variables $(\mathrm{WH}, \mathrm{BL}, \mathrm{CG}, \mathrm{CW}$ and PW)

Problem of multicollinearity was also considered using variance inflation factor (VIF) for each predictor in every age class (Eyduran et al., 2013; Eyduran et al., 2017; Chitra et al., 2012).

\section{RESULTS AND DISCUSSION}

Overall estimates of means of LWB, WH, BL, $\mathrm{CG}, \mathrm{CW}$ and $\mathrm{PW}$ are provided in Table 1 for each monthly age class. Live body weight and all body measurements displayed an increasing trend from birth to approximately 36 months of age, as expected. At birth, goats had average $( \pm \mathrm{SD}) \mathrm{LWB}, \mathrm{WH}, \mathrm{BL}, \mathrm{CG}, \mathrm{CW}$ and 
PW as $3.34 \pm 0.48,33.61 \pm 3.47,25.11 \pm 2.16,30.96 \pm 2.11$, $8.88 \pm 0.98$ and $4.69 \pm 0.97$, respectively. At weaning (4 months of age), goats had average ( \pm SD) LWB, WH, $\mathrm{BL}, \mathrm{CG}, \mathrm{CW}$ and $\mathrm{PW}$ as $18.84 \pm 3.71,64.54 \pm 4.24$, $54.53 \pm 3.96, \quad 59.85 \pm 3.75, \quad 17.06 \pm 2.37$ and 9.39 \pm 1.74 , respectively. Means values $( \pm \mathrm{SD})$ of $\mathrm{LWB}, \mathrm{WH}, \mathrm{BL}$, $\mathrm{CG}, \mathrm{CW}$ and PW at yearling were $33.47 \pm 4.83$, $76.75 \pm 4.43,66.15 \pm 3.66,73.24 \pm 4.07,20.59 \pm 2.78$ and $11.91 \pm 1.67$, respectively. The means values of all the handled quantitative traits under the present study are comparable to previously published data. However, the present study has, for the first time, reported on the monthly means of all traits up to 36 months of age.

Subsequently, the LBW was considered as a dependent/response variable with all body measurements traits as independent/explanatory variables and were analyzed in PROC GLM of SAS. Multicollinearity problem was also observed using variance inflation factor (VIF) for every predictor in each age class (Eyduran et al., 2013; Khan et al., 2014). The results of this analysis and the minimum to maximum values of VIF for each age class are reported in Table 2; moreover, with the exception of the first two and the last age classes, CG had highly significant impact on LWB at all ages proving the superiority of CG over all other body measurements in explaining the variation in LBW of goats. The PW has largely non-significant effect $(\mathrm{P} \leq 0.05)$ on LBW in most age classes up to 36 month of age suggesting that PW had little role in explaining variation in LBW of the Beetal goats. Other body measurement traits $\mathrm{WH}, \mathrm{BL}$ and $\mathrm{CW}$ showed intermediate impact on LBW i.e. between the effects of CG and PW. Regression coefficients for all the body measurements are presented in Table 3 for age classes 0 to 36 . The VIF and R-square are indicator of multicollinearity for multiple regression analysis. VIF is a measure of variance of a coefficient due to collinearity. The amount of VIF should be less than 10 for a reliable multiple linear regression analysis (Tariq et al., 2012; Eyduran et al., 2012). These equations can be implied to predict live body weight of the Beetal goats up to 36 months of age with modest accuracy.

The values of $\mathrm{R}$-square are also reported for the regression line in each age class in Table 3. R-squared is a statistical tool, which describes how close the data points are to the fitted regression line. It is more commonly called as coefficient of determination and may be defined as the coefficient of multiple determination for multiple regression as was the case in the present study. The range of $\mathrm{R}^{2}$ values for regression model is from 0.19 to 0.72 in various age groups. The value of $\mathrm{R}^{2}$ was lowest (0.19) for birth/0d class probably due to limited number of observations in this class. Overall value of $\mathrm{R}^{2}$ increased with increasing age and its highest value was obtained for animals aged as 17 months. With most $\mathrm{R}^{2}$ values above or around $0.50(50 \%)$ across 36 age classes, the proposed model seems to have adequately explained the variation in LBW of goats.

The relationship of body measurements and LBW was further studied using PROC CORR of SAS. Different body measurements showed significant and high positive correlation with LBW as well as with age (Table 4). The CG had strong positive/ highest correlation (0.93) with LBW. The PW also was positively correlated with LBW (0.74) but magnitude was lowest for pairs of all traits under the study. These results were in accordance with the results of other previous studies (Adeyinka and Mohammed, 2006; Otoikhian et al., 2008; Moela, 2014). Therefore, CG has been found the most significant and important measure to estimate the live body weight of Beetal at any age of the animal. $\mathrm{PW}$ is found to be non-significant and with negative regression coefficients parameters values in many of the age groups.

In a previous study by Iqbal et al., in 2013 based on 230 female Beetal goats, comparable $\mathrm{R}^{2}$ values $(69.1 \%)$ for a model having $\mathrm{BL}$ and $\mathrm{WH}$ body measurements as predictors. In agreement to the results of the present study, CG had been reported as a significant predictor of LBW in Afar, Saanen and Beetal breeds of goat, respectively (Seifemichael et al., 2014; Pesmen and Yardimci, 2008; Iqbal et al., 2013). The correlation coefficient of LBW and $\mathrm{CG}$ in the present study was comparable with the results reported by Fahim et al. (2013) by developing age classes to study the effects of different body measurements in Rohilkhand goats. It was reported that the most significant LBW predictors were sex, rump height, shank circumference and head girth in Beetal goats (Eyduran et al., 2017). Iqbal et al. (2013) reported $\mathrm{R}^{2}$ values ranging from 0.16 to 0.69 using different body measurements in Beetal goats with the maximum value reported was for $\mathrm{WH}$ and CG. $\mathrm{R}^{2}$ of linear regression model was 0.85 and comparable $\mathrm{R}^{2}$ value 0.72 for regression model using $\mathrm{BL}$, $\mathrm{WH}$ and CG body measurements as LBW predictors was reported for Malabari goats by Chitra et al. (2012). The similar values of VIF were reported by Eyduran et al. (2013) who used the comparable body measurements for commercial goats (crossbred of the Beetal breed). 
Table 1. Means \pm S.D. of live body weight (kg) and body measurements (cm)

\begin{tabular}{|c|c|c|c|c|c|c|c|}
\hline $\begin{array}{l}\text { Age groups } \\
\text { (months) }\end{array}$ & $\mathbf{N}$ & $\begin{array}{c}\text { Live body } \\
\text { weight }\end{array}$ & $\begin{array}{c}\text { Whither } \\
\text { height }\end{array}$ & $\begin{array}{l}\text { Body } \\
\text { length }\end{array}$ & Chest girth & $\begin{array}{l}\text { Chest } \\
\text { width }\end{array}$ & $\begin{array}{c}\text { Pin bone } \\
\text { width }\end{array}$ \\
\hline 0 (Birth) & 26 & $3.34 \pm 0.48$ & $33.61 \pm 3.47$ & $25.11 \pm 2.16$ & $30.96 \pm 2.11$ & $8.88 \pm 0.98$ & $4.69 \pm 0.97$ \\
\hline 1 & 108 & $8.31 \pm 2.33$ & $48.82 \pm 6.99$ & $40.41 \pm 5.25$ & $44.97 \pm 5.65$ & $12.14 \pm 2.78$ & $6.67 \pm 1.93$ \\
\hline 2 & 153 & $13.00 \pm 2.50$ & $55.95 \pm 5.37$ & $46.80 \pm 5.46$ & $52.14 \pm 6.19$ & $14.21 \pm 2.48$ & $7.67 \pm 2.00$ \\
\hline 3 & 145 & $17.57 \pm 3.82$ & $61.92 \pm 5.09$ & $52.08 \pm 4.08$ & $57.40 \pm 3.98$ & $16.04 \pm 2.39$ & $8.59 \pm 1.70$ \\
\hline 4 & 150 & $18.84 \pm 3.71$ & $64.54 \pm 4.24$ & $54.53 \pm 3.96$ & $59.85 \pm 3.75$ & $17.06 \pm 2.37$ & $9.39 \pm 1.74$ \\
\hline 5 & 109 & $21.32 \pm 3.61$ & $66.62 \pm 4.73$ & $56.77 \pm 3.68$ & $62.56 \pm 4.50$ & $17.78 \pm 2.44$ & $9.91 \pm 1.57$ \\
\hline 6 & 146 & $21.90 \pm 3.96$ & $68.32 \pm 4.74$ & $58.13 \pm 4.13$ & $63.35 \pm 4.26$ & $18.14 \pm 2.14$ & $10.00 \pm 1.52$ \\
\hline 7 & 145 & $23.59 \pm 3.91$ & $69.70 \pm 4.21$ & $59.40 \pm 3.73$ & $65.41 \pm 4.16$ & $18.78 \pm 2.20$ & $10.40 \pm 1.48$ \\
\hline 8 & 171 & $25.26 \pm 3.31$ & $71.26 \pm 3.60$ & $60.94 \pm 3.29$ & $67.50 \pm 3.71$ & $19.01 \pm 2.52$ & $10.65 \pm 1.55$ \\
\hline 9 & 177 & $27.75 \pm 4.54$ & $72.50 \pm 4.28$ & $62.59 \pm 3.63$ & $69.11 \pm 4.22$ & $19.99 \pm 2.01$ & $11.14 \pm 1.35$ \\
\hline 10 & 212 & $29.85 \pm 4.76$ & $74.21 \pm 4.06$ & $63.94 \pm 3.59$ & $70.52 \pm 3.97$ & $19.98 \pm 2.29$ & $11.21 \pm 1.38$ \\
\hline 11 & 261 & $30.87 \pm 4.40$ & $74.67 \pm 3.74$ & $64.64 \pm 3.31$ & $71.44 \pm 3.67$ & $20.46 \pm 2.15$ & $11.73 \pm 1.44$ \\
\hline 12 & 297 & $33.47 \pm 4.83$ & $76.75 \pm 4.43$ & $66.15 \pm 3.66$ & $73.24 \pm 4.07$ & $20.59 \pm 2.78$ & $11.91 \pm 1.67$ \\
\hline 13 & 199 & $36.13 \pm 4.55$ & $77.75 \pm 3.71$ & $67.60 \pm 3.22$ & $75.26 \pm 4.02$ & $21.50 \pm 2.12$ & $12.54 \pm 1.45$ \\
\hline 14 & 223 & $37.81 \pm 5.15$ & $79.29 \pm 3.77$ & $68.56 \pm 3.30$ & $76.26 \pm 3.86$ & $21.72 \pm 2.61$ & $12.64 \pm 1.84$ \\
\hline 15 & 172 & $40.28 \pm 5.43$ & $80.47 \pm 4.14$ & $69.58 \pm 3.52$ & $78.36 \pm 3.81$ & $22.33 \pm 2.60$ & $12.78 \pm 1.68$ \\
\hline 16 & 175 & $40.56 \pm 5.64$ & $80.48 \pm 4.24$ & $69.62 \pm 3.80$ & $78.75 \pm 3.95$ & $22.40 \pm 2.21$ & $13.22 \pm 1.45$ \\
\hline 17 & 152 & $42.27 \pm 5.90$ & $81.26 \pm 3.88$ & $70.62 \pm 3.46$ & $79.51 \pm 4.33$ & $22.52 \pm 2.26$ & $13.34 \pm 1.72$ \\
\hline 18 & 149 & $42.79 \pm 6.27$ & $82.34 \pm 3.82$ & $71.57 \pm 3.79$ & $79.93 \pm 4.11$ & $22.33 \pm 2.77$ & $13.23 \pm 1.96$ \\
\hline 19 & 121 & $43.32 \pm 5.69$ & $82.88 \pm 4.01$ & $71.45 \pm 3.97$ & $80.68 \pm 4.05$ & $23.01 \pm 2.35$ & $13.74 \pm 1.77$ \\
\hline 20 & 157 & $42.64 \pm 6.11$ & $82.91 \pm 4.06$ & $71.40 \pm 3.64$ & $80.28 \pm 3.89$ & $23.02 \pm 2.75$ & $13.61 \pm 1.86$ \\
\hline 21 & 107 & $42.66 \pm 6.16$ & $82.35 \pm 4.18$ & $71.85 \pm 3.52$ & $80.59 \pm 3.80$ & $22.89 \pm 2.44$ & $13.50 \pm 1.71$ \\
\hline 22 & 108 & $44.99 \pm 7.26$ & $82.78 \pm 6.02$ & $72.51 \pm 3.30$ & $81.27 \pm 4.21$ & $22.82 \pm 2.61$ & $13.26 \pm 1.86$ \\
\hline 23 & 132 & $46.20 \pm 6.20$ & $83.17 \pm 3.32$ & $72.64 \pm 3.04$ & $81.91 \pm 3.68$ & $22.91 \pm 2.60$ & $13.64 \pm 1.74$ \\
\hline 24 & 136 & $47.06 \pm 7.36$ & $84.13 \pm 3.79$ & $73.85 \pm 4.13$ & $82.50 \pm 4.71$ & $22.72 \pm 3.26$ & $12.98 \pm 2.40$ \\
\hline 25 & 140 & $48.96 \pm 7.76$ & $85.12 \pm 4.91$ & $73.92 \pm 4.25$ & $83.71 \pm 5.25$ & $24.11 \pm 2.82$ & $14.40 \pm 1.95$ \\
\hline 26 & 109 & $49.63 \pm 7.21$ & $85.05 \pm 4.55$ & $74.54 \pm 3.74$ & $83.80 \pm 4.63$ & $23.58 \pm 3.24$ & $14.08 \pm 2.19$ \\
\hline 27 & 83 & $49.60 \pm 6.08$ & $84.31 \pm 4.94$ & $74.59 \pm 3.95$ & $84.27 \pm 5.14$ & $23.57 \pm 3.46$ & $13.75 \pm 2.50$ \\
\hline 28 & 83 & $53.13 \pm 6.92$ & $85.45 \pm 4.07$ & $75.26 \pm 3.87$ & $85.54 \pm 4.19$ & $24.36 \pm 3.11$ & $14.30 \pm 2.21$ \\
\hline 29 & 68 & $56.98 \pm 7.11$ & $86.25 \pm 4.65$ & $75.79 \pm 3.73$ & $86.69 \pm 4.43$ & $23.27 \pm 3.09$ & $13.96 \pm 2.55$ \\
\hline 30 & 94 & $56.14 \pm 9.14$ & $86.37 \pm 5.76$ & $76.85 \pm 4.70$ & $86.30 \pm 5.38$ & $23.47 \pm 3.33$ & $13.27 \pm 2.88$ \\
\hline 31 & 56 & $58.48 \pm 8.31$ & $87.26 \pm 5.80$ & $76.60 \pm 5.28$ & $88.35 \pm 5.45$ & $24.26 \pm 2.89$ & $14.97 \pm 2.83$ \\
\hline 32 & 65 & $61.32 \pm 9.08$ & $87.89 \pm 5.27$ & $77.87 \pm 4.82$ & $89.10 \pm 4.91$ & $25.13 \pm 2.64$ & $15.04 \pm 2.70$ \\
\hline 33 & 66 & $63.91 \pm 10.61$ & $87.47 \pm 4.96$ & $77.66 \pm 4.64$ & $90.36 \pm 5.15$ & $25.31 \pm 2.95$ & $14.93 \pm 2.56$ \\
\hline 34 & 66 & $65.86 \pm 11.99$ & $88.00 \pm 5.61$ & $78.10 \pm 5.48$ & $90.89 \pm 6.14$ & $26.79 \pm 3.40$ & $15.87 \pm 2.28$ \\
\hline 35 & 227 & $65.83 \pm 10.49$ & $89.03 \pm 5.35$ & $79.22 \pm 5.66$ & $90.97 \pm 5.60$ & $26.72 \pm 3.64$ & $15.65 \pm 2.40$ \\
\hline 36 & 23 & $80.21 \pm 16.79$ & $92.91 \pm 8.45$ & $84.86 \pm 6.69$ & $93.30 \pm 8.24$ & $26.21 \pm 5.25$ & $14.00 \pm 3.71$ \\
\hline Total & 5011 & & & & & & \\
\hline
\end{tabular}

Table 2. Effect of body measurements on body weight in the Beetal goats

\begin{tabular}{|c|c|c|c|c|c|c|c|}
\hline \multirow{2}{*}{$\begin{array}{l}\text { Age groups } \\
\text { (months) }\end{array}$} & \multirow[t]{2}{*}{$\mathbf{N}$} & \multicolumn{6}{|c|}{ Probability values for tests of significance (F-test) from PROC GLM of SAS } \\
\hline & & $\begin{array}{c}\text { Whither } \\
\text { height }\end{array}$ & Body length & $\begin{array}{l}\text { Chest } \\
\text { girth }\end{array}$ & Chest width & $\begin{array}{l}\text { Pin bone } \\
\text { width }\end{array}$ & $\begin{array}{l}\text { VIF value } \\
\text { (Min-Max) }\end{array}$ \\
\hline 0 (Birth) & 26 & $0.4610^{\mathrm{NS1}}$ & $0.2524^{\mathrm{NS}}$ & $0.8069^{\mathrm{NS}}$ & $0.4865^{\mathrm{NS}}$ & $0.7851^{\mathrm{NS}}$ & $1.18-1.76$ \\
\hline 1 & 108 & $0.1511^{\mathrm{NS}}$ & $\leq .0001^{* * * 2}$ & $0.0640^{\mathrm{NS}}$ & $0.9411^{\mathrm{NS}}$ & $0.2328^{\mathrm{NS}}$ & 2.16-3.04 \\
\hline 2 & 153 & $0.0589^{\mathrm{NS}}$ & $0.1403^{\mathrm{NS}}$ & $0.1194^{\mathrm{NS}}$ & $0.1935^{\mathrm{NS}}$ & $0.2664^{\mathrm{NS}}$ & $2.05-3.26$ \\
\hline 3 & 145 & $0.0008 * *$ & $0.0463 *$ & $0.0015^{* *}$ & $0.0047 * *$ & $\leq .0001 * * *$ & $1.62-3.06$ \\
\hline 4 & 150 & $0.7817^{\mathrm{NS}}$ & $\leq .0001 * * *$ & $0.0013 * *$ & $0.0300 *$ & $0.0148 *$ & $2.00-3.14$ \\
\hline 5 & 109 & $0.0674^{\mathrm{NS}}$ & $0.0028 * *$ & $0.0105^{*}$ & $0.1641^{\mathrm{NS}}$ & $0.9233^{\mathrm{NS}}$ & $1.80-3.55$ \\
\hline 6 & 146 & $0.1730^{\mathrm{NS}}$ & $\leq .0001 * * *$ & $0.0054 * *$ & $0.0025 * *$ & $0.2686^{\mathrm{NS}}$ & 1.96-4.19 \\
\hline 7 & 145 & $0.0390 *$ & $0.0174 *$ & $0.0737^{\mathrm{NS}}$ & $0.0027 * *$ & $0.9801^{\mathrm{NS}}$ & 1.71-3.22 \\
\hline
\end{tabular}




\begin{tabular}{|c|c|c|c|c|c|c|c|}
\hline 8 & 171 & $0.5396^{\mathrm{NS}}$ & $0.0364 *$ & $\leq .0001 * * *$ & $0.1150^{\mathrm{NS}}$ & $0.8087^{\mathrm{NS}}$ & $1.46-1.83$ \\
\hline 9 & 177 & $0.1436^{\mathrm{NS}}$ & $0.1294^{\mathrm{NS}}$ & $\leq .0001 * * *$ & $0.7264^{\mathrm{NS}}$ & $0.0041 * *$ & $1.43-2.69$ \\
\hline 10 & 212 & $\leq .0001^{* * *}$ & $0.0661^{\mathrm{NS}}$ & $\leq .0001 * * *$ & $0.1145^{\mathrm{NS}}$ & $0.0020 * *$ & $1.62-2.73$ \\
\hline 11 & 261 & $\leq .0001 * * *$ & $0.1173^{\mathrm{NS}}$ & $\leq .0001 * * *$ & $0.1770^{\mathrm{NS}}$ & $\leq .0001 * * *$ & $1.55-2.30$ \\
\hline 12 & 297 & $\leq .0001 * * *$ & $\leq .0001 * * *$ & $\leq .0001 * * *$ & $0.0032 * *$ & $0.8888^{\mathrm{NS}}$ & $1.35-\mathbf{2 . 0 6}$ \\
\hline 13 & 199 & $0.6812^{\mathrm{NS}}$ & $0.0036^{* *}$ & $\leq .0001 * * *$ & $0.0454 *$ & $0.4876^{\mathrm{NS}}$ & $1.41-2.06$ \\
\hline 14 & 223 & $0.0053^{*}$ & $\leq .0001 * * *$ & $\leq .0001 * * *$ & $0.0021 * *$ & $0.1353^{\mathrm{NS}}$ & $1.44-2.66$ \\
\hline 15 & 172 & $\leq .0001 * * *$ & $\leq .0001 * * *$ & $0.0008^{* *}$ & $\leq .0001 * * *$ & $0.5453^{\mathrm{NS}}$ & $1.28-2.85$ \\
\hline 16 & 175 & $\leq .0001 * * *$ & $0.1005^{\mathrm{NS}}$ & $\leq .0001 * * *$ & $0.0031 * *$ & $0.6274^{\mathrm{NS}}$ & $1.41-2.62$ \\
\hline 17 & 152 & $\leq .0001 * * *$ & $0.0186^{*}$ & $\leq .0001 * * *$ & $0.0427 *$ & $0.2657^{\mathrm{NS}}$ & $2.01-2.22$ \\
\hline 18 & 149 & $0.0669^{\mathrm{NS}}$ & $\leq .0001 * * *$ & $\leq .0001 * * *$ & $0.0151 *$ & $0.1881^{\mathrm{NS}}$ & $1.33-2.97$ \\
\hline 19 & 121 & $0.1421^{\mathrm{NS}}$ & $0.0205^{*}$ & $\leq .0001 * * *$ & $0.1340^{\mathrm{NS}}$ & $0.9694^{\mathrm{NS}}$ & $1.99-2.44$ \\
\hline 20 & 157 & $0.0022^{* *}$ & $0.0664^{\mathrm{NS}}$ & $\leq .0001 * * *$ & $0.0052 * *$ & $0.5643^{\mathrm{NS}}$ & $2.05-3.48$ \\
\hline 21 & 107 & $0.0294 *$ & $0.0010 * *$ & $\leq .0001 * * *$ & $0.0051 * *$ & $0.0280 *$ & $1.50-2.78$ \\
\hline 22 & 108 & $0.0214 *$ & $0.2098^{\mathrm{NS}}$ & $\leq .0001 * * *$ & $0.4698^{\mathrm{NS}}$ & $0.5316^{\mathrm{NS}}$ & $1.68-2.92$ \\
\hline 23 & 132 & $0.6669^{\mathrm{NS}}$ & $0.1329^{\mathrm{NS}}$ & $\leq .0001 * * *$ & $0.9879^{\mathrm{NS}}$ & $0.7008^{\mathrm{NS}}$ & $1.33-2.41$ \\
\hline 24 & 136 & $0.9606^{\mathrm{NS}}$ & $0.0085^{* *}$ & $\leq .0001 * * *$ & $0.0981^{\mathrm{NS}}$ & $0.7442^{\mathrm{NS}}$ & $1.59-2.64$ \\
\hline 25 & 140 & $0.4415^{\mathrm{NS}}$ & $0.0278^{*}$ & $\leq .0001 * * *$ & $0.5618^{\mathrm{NS}}$ & $0.7022^{\mathrm{NS}}$ & $2.06-3.73$ \\
\hline 26 & 109 & $0.4706^{\mathrm{NS}}$ & $0.0032 * *$ & $\leq .0001 * * *$ & $0.7129^{\mathrm{NS}}$ & $0.4006^{\mathrm{NS}}$ & $1.67-2.97$ \\
\hline 27 & 83 & $0.4603^{\mathrm{NS}}$ & $0.8040^{\mathrm{NS}}$ & $0.0005^{* *}$ & $0.1140^{\mathrm{NS}}$ & $0.1137^{\mathrm{NS}}$ & $1.70-4.26$ \\
\hline 28 & 83 & $0.2560^{\mathrm{NS}}$ & $0.8501^{\mathrm{NS}}$ & $\leq .0001 * * *$ & $0.9549^{\mathrm{NS}}$ & $0.2528^{\mathrm{NS}}$ & $1.53-2.49$ \\
\hline 29 & 68 & $0.8885^{\mathrm{NS}}$ & $0.3650^{\mathrm{NS}}$ & $\leq .0001 * * *$ & $0.8269^{\mathrm{NS}}$ & $0.2659^{\mathrm{NS}}$ & $1.37-2.25$ \\
\hline 30 & 94 & $0.4704^{\mathrm{NS}}$ & $0.6975^{\mathrm{NS}}$ & $0.0001 * *$ & $0.0349 *$ & $0.0578^{\mathrm{NS}}$ & $1.45-3.56$ \\
\hline 31 & 56 & $0.3304^{\mathrm{NS}}$ & $0.9057^{\mathrm{NS}}$ & $0.0005^{* *}$ & $0.7231^{\mathrm{NS}}$ & $0.7131^{\mathrm{NS}}$ & $1.34-3.11$ \\
\hline 32 & 65 & $0.4720^{\mathrm{NS}}$ & $0.2847^{\mathrm{NS}}$ & $0.0031 * *$ & $0.0305 *$ & $0.0062 * *$ & $1.45-2.44$ \\
\hline 33 & 66 & $0.1351^{\mathrm{NS}}$ & $0.7964^{\mathrm{NS}}$ & $\leq .0001 * * *$ & $0.0951^{\mathrm{NS}}$ & $0.1489^{\mathrm{NS}}$ & $1.69-2.39$ \\
\hline 34 & 66 & $0.0622^{\mathrm{NS}}$ & $0.7433^{\mathrm{NS}}$ & $\leq .0001 * * *$ & $0.8635^{\mathrm{NS}}$ & $0.7191^{\mathrm{NS}}$ & $2.10-3.31$ \\
\hline 35 & 227 & $0.0178 *$ & $0.9516^{\mathrm{NS}}$ & $\leq .0001 * * *$ & $\leq .0001 * * *$ & $0.0042 * *$ & $1.82-2.82$ \\
\hline 36 & 23 & $0.8582^{\mathrm{NS}}$ & $0.3892^{\mathrm{NS}}$ & $0.0575^{\mathrm{NS}}$ & $0.3236^{\mathrm{NS}}$ & $0.9099^{\mathrm{NS}}$ & $2.20-7.75$ \\
\hline Overall & 5011 & $0.051^{\mathrm{NS}}$ & $0.000 * *$ & $0.000 * *$ & $0.000 *$ & $0.264^{\mathrm{NS}}$ & $4.31-9.21$ \\
\hline
\end{tabular}

${ }^{1} \mathrm{NS}=$ Non-significant

$2 *=$ Probability values $(* \mathrm{P} \leq 0.05, * * \mathrm{P} \leq 0.01, * * * \mathrm{P} \leq 0.001)$ show significance

Table 3. Parameter \pm S.E. estimates of different body measurements to predict body weight

\begin{tabular}{|c|c|c|c|c|c|c|c|}
\hline $\begin{array}{c}\text { Age } \\
\text { groups }\end{array}$ & Intercept & $\begin{array}{c}\text { Whither } \\
\text { height }\end{array}$ & Body length & Chest girth & Chest width & $\begin{array}{l}\text { Pin bone } \\
\text { width }\end{array}$ & $\begin{array}{c}\mathbf{R}^{2} \\
\text { Value }\end{array}$ \\
\hline 0 & $-0.23 \pm 2.08^{\mathrm{NSI}}$ & $0.03 \pm 0.04^{\mathrm{NS}}$ & $0.06 \pm 0.05^{\mathrm{NS}}$ & $0.01 \pm 0.05^{\mathrm{NS}}$ & $0.09 \pm 0.13^{\mathrm{NS}}$ & $-0.04 \pm 0.13^{\mathrm{NS}}$ & 0.187 \\
\hline 1 & $-5.46 \pm 1.47 *^{2}$ & $0.06 \pm 0.04^{\mathrm{NS}}$ & $0.19 \pm 0.05^{*}$ & $0.09 \pm 0.05^{\mathrm{NS}}$ & $-0.01 \pm 0.10^{\mathrm{NS}}$ & $-0.17 \pm 0.14^{\mathrm{NS}}$ & 0.477 \\
\hline 2 & $-1.11 \pm 1.81^{\mathrm{NS}}$ & $0.09 \pm 0.05^{\mathrm{NS}}$ & $0.08 \pm 0.06^{\mathrm{NS}}$ & $0.08 \pm 0.05^{\mathrm{NS}}$ & $0.14 \pm 0.11^{\mathrm{NS}}$ & $-0.14 \pm 0.12^{\mathrm{NS}}$ & 0.315 \\
\hline 3 & $-19.84 \pm 3.23 *$ & $0.25 \pm 0.07 *$ & $0.18 \pm 0.09^{*}$ & $0.24 \pm 0.07 *$ & $0.34 \pm 0.12 *$ & $-0.77 \pm 0.16^{*}$ & 0.574 \\
\hline 4 & $-22.72 \pm 3.61 *$ & $0.02 \pm 0.09^{\mathrm{NS}}$ & $0.38 \pm 0.09 *$ & $0.31 \pm 0.09^{*}$ & $0.28 \pm 0.13 *$ & $-0.41 \pm 0.17^{*}$ & 0.551 \\
\hline 5 & $-24.03 \pm 3.91 *$ & $0.17 \pm 0.09^{\mathrm{NS}}$ & $0.30 \pm 0.10^{*}$ & $0.22 \pm 0.09^{*}$ & $0.18 \pm 0.13^{\mathrm{NS}}$ & $0.02 \pm 0.20^{\mathrm{NS}}$ & 0.579 \\
\hline 6 & $-28.85 \pm 3.42 *$ & $0.12 \pm 0.09^{\mathrm{NS}}$ & $0.36 \pm 0.09 *$ & $0.24 \pm 0.09 *$ & $0.46 \pm 0.15^{*}$ & $-0.21 \pm 0.19^{\mathrm{NS}}$ & 0.633 \\
\hline 7 & $-26.19 \pm 4.46^{*}$ & $0.21 \pm 0.10^{*}$ & $0.24 \pm 0.10^{*}$ & $0.18 \pm 0.10^{\mathrm{NS}}$ & $0.47 \pm 0.15^{*}$ & $-0.01 \pm 0.21^{\mathrm{NS}}$ & 0.480 \\
\hline 8 & $-13.76 \pm 4.73 *$ & $0.05 \pm 0.08^{\mathrm{NS}}$ & $0.18 \pm 0.09 *$ & $0.31 \pm 0.08^{*}$ & $0.17 \pm 0.11^{\mathrm{NS}}$ & $0.04 \pm 0.16^{\mathrm{NS}}$ & 0.328 \\
\hline 9 & $-33.40 \pm 4.77 *$ & $0.13 \pm 0.09^{\mathrm{NS}}$ & $0.13 \pm 0.09^{\mathrm{NS}}$ & $0.52 \pm 0.09 *$ & $0.05 \pm 0.14^{\mathrm{NS}}$ & $0.60 \pm 0.21 *$ & 0.541 \\
\hline 10 & $-48.05 \pm 4.59 *$ & $0.41 \pm 0.08^{*}$ & $0.14 \pm 0.08^{\mathrm{NS}}$ & $0.40 \pm 0.09 *$ & $0.19 \pm 0.12^{\mathrm{NS}}$ & $0.60 \pm 0.19 *$ & 0.601 \\
\hline 11 & $-44.47 \pm 4.27^{*}$ & $0.37 \pm 0.07^{*}$ & $0.11 \pm 0.07^{\mathrm{NS}}$ & $0.41 \pm 0.07^{*}$ & $0.15 \pm 0.11^{\mathrm{NS}}$ & $0.73 \pm 0.16^{*}$ & 0.585 \\
\hline 12 & $-46.51 \pm 4.33^{*}$ & $0.36 \pm 0.06^{*}$ & $0.37 \pm 0.06^{*}$ & $0.31 \pm 0.07^{*}$ & $0.27 \pm 0.09^{*}$ & $-0.02 \pm 0.15^{\mathrm{NS}}$ & 0.547 \\
\hline 13 & $-37.66 \pm 5.80 *$ & $-0.03 \pm 0.07^{\mathrm{NS}}$ & $0.23 \pm 0.08 *$ & $0.71 \pm 0.07 *$ & $0.25 \pm 0.13 *$ & $0.12 \pm 0.17^{\mathrm{NS}}$ & 0.592 \\
\hline 14 & $-49.92 \pm 6.19 *$ & $0.23 \pm 0.08 *$ & $0.35 \pm 0.09^{*}$ & $0.51 \pm 0.08^{*}$ & $0.47 \pm 0.15^{*}$ & $-0.30 \pm 0.20^{\mathrm{NS}}$ & 0.520 \\
\hline 15 & $-63.99 \pm 6.69 *$ & $0.35 \pm 0.08^{*}$ & $0.48 \pm 0.08^{*}$ & $0.33 \pm 0.10^{*}$ & $0.66 \pm 0.17 *$ & $0.15 \pm 0.25^{\mathrm{NS}}$ & 0.631 \\
\hline 16 & $-59.14 \pm 6.28 *$ & $0.49 \pm 0.09 *$ & $0.14 \pm 0.08^{\mathrm{NS}}$ & $0.51 \pm 0.11^{*}$ & $0.54 \pm 0.18^{*}$ & $-0.13 \pm 0.27^{\mathrm{NS}}$ & 0.621 \\
\hline 17 & $-64.84 \pm 6.23 *$ & $0.42 \pm 0.10 *$ & $0.26 \pm 0.11^{*}$ & $0.55 \pm 0.09 *$ & $0.35 \pm 0.17 *$ & $0.25 \pm 0.23^{\mathrm{NS}}$ & 0.708 \\
\hline 18 & $-70.82 \pm 7.87 *$ & $0.24 \pm 0.13^{\mathrm{NS}}$ & $0.41 \pm 0.10^{*}$ & $0.73 \pm 0.12 *$ & $0.50 \pm 0.20^{*}$ & $-0.37 \pm 0.28^{\mathrm{NS}}$ & 0.620 \\
\hline
\end{tabular}




\begin{tabular}{|c|c|c|c|c|c|c|c|}
\hline 19 & $-53.48 \pm 7.17 *$ & $0.18 \pm 0.12^{\mathrm{NS}}$ & $0.29 \pm 0.12 *$ & $0.67 \pm 0.12 *$ & $0.30 \pm 0.20^{\mathrm{NS}}$ & $0.01 \pm 0.25^{\mathrm{NS}}$ & 0.640 \\
\hline 20 & $-62.50 \pm 7.21 *$ & $0.33 \pm 0.11 *$ & $0.22 \pm 0.12^{\mathrm{NS}}$ & $0.65 \pm 0.12 *$ & $0.56 \pm 0.20 *$ & $-0.17 \pm 0.30^{\mathrm{NS}}$ & 0.646 \\
\hline 21 & $-70.72 \pm 9.45^{*}$ & $0.25 \pm 0.11 *$ & $0.46 \pm 0.13^{*}$ & $0.67 \pm 0.14^{*}$ & $0.74 \pm 0.26^{*}$ & $-0.79 \pm 0.35^{*}$ & 0.613 \\
\hline 22 & $-67.23 \pm 12.06^{*}$ & $0.24 \pm 0.10 *$ & $0.20 \pm 0.16^{\mathrm{NS}}$ & $0.93 \pm 0.16^{*}$ & $0.23 \pm 0.32^{\mathrm{NS}}$ & $-0.26 \pm 0.42^{\mathrm{NS}}$ & 0.551 \\
\hline 23 & $-34.69 \pm 13.62 *$ & $-0.07 \pm 0.16^{\mathrm{NS}}$ & $0.31 \pm 0.20^{\mathrm{NS}}$ & $0.81 \pm 0.16^{*}$ & $-0.01 \pm 0.27^{\mathrm{NS}}$ & $-0.16 \pm 0.40^{\mathrm{NS}}$ & 0.313 \\
\hline 24 & $-54.85 \pm 10.50 *$ & $-0.01 \pm 0.14^{\mathrm{NS}}$ & $0.38 \pm 0.14^{*}$ & $0.82 \pm 0.14^{*}$ & $0.35 \pm 0.21^{\mathrm{NS}}$ & $-0.10 \pm 0.29^{\mathrm{NS}}$ & 0.556 \\
\hline 25 & $-45.27 \pm 9.52 *$ & $-0.15 \pm 0.19^{\mathrm{NS}}$ & $0.47 \pm 0.21 *$ & $0.88 \pm 0.14^{*}$ & $-0.17 \pm 0.29^{\mathrm{NS}}$ & $0.14 \pm 0.35^{\mathrm{NS}}$ & 0.487 \\
\hline 26 & $-68.08 \pm 10.39 *$ & $0.10 \pm 0.14^{\mathrm{NS}}$ & $0.46 \pm 0.15^{*}$ & $0.87 \pm 0.14^{*}$ & $-0.09 \pm 0.24^{\mathrm{NS}}$ & $0.27 \pm 0.32^{\mathrm{NS}}$ & 0.612 \\
\hline 27 & $-10.92 \pm 12.32^{\mathrm{NS}}$ & $0.11 \pm 0.143^{\mathrm{NS}}$ & $-0.04 \pm 0.18^{\mathrm{NS}}$ & $0.61 \pm 0.17 *$ & $0.51 \pm 0.32^{\mathrm{NS}}$ & $-0.60 \pm 0.37^{\mathrm{NS}}$ & 0.409 \\
\hline 28 & $-55.96 \pm 13.68^{*}$ & $0.19 \pm 0.17^{\mathrm{NS}}$ & $0.03 \pm 0.18^{\mathrm{NS}}$ & $0.98 \pm 0.17^{*}$ & $0.02 \pm 0.28^{\mathrm{NS}}$ & $0.42 \pm 0.37^{\mathrm{NS}}$ & 0.523 \\
\hline 29 & $-43.46 \pm 16.56^{*}$ & $-0.03 \pm 0.19^{\mathrm{NS}}$ & $0.19 \pm 0.21^{\mathrm{NS}}$ & $0.93 \pm 0.20 *$ & $0.07 \pm 0.32^{\mathrm{NS}}$ & $0.40 \pm 0.36^{\mathrm{NS}}$ & 0.452 \\
\hline 30 & $-43.80 \pm 13.46^{*}$ & $0.13 \pm 0.18^{\mathrm{NS}}$ & $0.07 \pm 0.18^{\mathrm{NS}}$ & $0.85 \pm 0.21^{*}$ & $0.84 \pm 0.39 *$ & $-0.75 \pm 0.39^{\mathrm{NS}}$ & 0.492 \\
\hline 31 & $-42.40 \pm 15.94^{*}$ & $0.21 \pm 0.21^{\mathrm{NS}}$ & $0.02 \pm 0.18^{\mathrm{NS}}$ & $0.84 \pm 0.22 *$ & $0.18 \pm 0.50^{\mathrm{NS}}$ & $0.19 \pm 0.50^{\mathrm{NS}}$ & 0.512 \\
\hline 32 & $-51.91 \pm 17.87^{*}$ & $0.18 \pm 0.24^{\mathrm{NS}}$ & $0.23 \pm 0.21^{\mathrm{NS}}$ & $0.82 \pm 0.27^{*}$ & $0.97 \pm 0.43^{*}$ & $-1.16 \pm 0.41 *$ & 0.497 \\
\hline 33 & $-76.88 \pm 21.06^{*}$ & $0.46 \pm 0.31^{\mathrm{NS}}$ & $-0.07 \pm 0.27^{\mathrm{NS}}$ & $1.28 \pm 0.26^{*}$ & $-0.83 \pm 0.49^{\mathrm{NS}}$ & $0.78 \pm 0.53^{\mathrm{NS}}$ & 0.489 \\
\hline 34 & $-81.54 \pm 17.24^{*}$ & $0.52 \pm 0.27^{\mathrm{NS}}$ & $-0.10 \pm 0.30^{\mathrm{NS}}$ & $1.22 \pm 0.28 *$ & $0.09 \pm 0.53^{\mathrm{NS}}$ & $-0.23 \pm 0.62^{\mathrm{NS}}$ & 0.597 \\
\hline 35 & $-57.32 \pm 8.54^{*}$ & $0.27 \pm 0.11 *$ & $0.01 \pm 0.10^{\mathrm{NS}}$ & $0.88 \pm 0.13^{*}$ & $1.14 \pm 0.20^{*}$ & $-0.78 \pm 0.27 *$ & 0.609 \\
\hline 36 & $-40.76 \pm 30.33^{\mathrm{NS}}$ & $-0.10 \pm 0.52^{\mathrm{NS}}$ & $-0.43 \pm 0.48^{\mathrm{NS}}$ & $1.49 \pm 0.73^{\mathrm{NS}}$ & $1.09 \pm 1.08^{\mathrm{NS}}$ & $-0.15 \pm 1.35^{\mathrm{NS}}$ & 0.715 \\
\hline overall & $-54.52 \pm 0.65^{*}$ & $-0.05 \pm 0.03^{\mathrm{NS}}$ & $0.19 \pm 0.03 *$ & $1.03 \pm 0.03 *$ & $0.35 \pm 0.05^{*}$ & $-0.07 \pm 0.06^{\mathrm{NS}}$ & 0.851 \\
\hline
\end{tabular}

Table 4. Estimates of Pearson Correlation Coefficients of body weight, age and different body measurements

\begin{tabular}{|c|c|c|c|c|c|c|c|}
\hline & Age & LBW & WH & $\mathrm{BL}$ & $\mathrm{CG}$ & $\mathrm{CW}$ & PW \\
\hline Age & 1 & $0.907 *^{1}$ & $0.802 *$ & $0.829 *$ & $0.863 *$ & $0.705^{*}$ & $0.687 *$ \\
\hline LBW & & 1 & $0.864 *$ & $0.875^{*}$ & $0.921 *$ & $0.777^{*}$ & $0.725^{*}$ \\
\hline WH & & & 1 & $0.924 *$ & $0.938 *$ & $0.754 *$ & $0.709 *$ \\
\hline BL & & & & 1 & $0.935 *$ & $0.771 *$ & $0.728 *$ \\
\hline $\mathrm{CG}$ & & & & & 1 & $0.811^{*}$ & $0.766^{*}$ \\
\hline $\mathrm{CW}$ & & & & & & 1 & $0.870 *$ \\
\hline PW & & & & & & & 1 \\
\hline
\end{tabular}

$\mathrm{LBW}=$ Live bodyweight, $\mathrm{WH}=$ Wither height, $\mathrm{BL}=$ Body length, $\mathrm{CG}=\mathrm{Chest}$ girth

$\mathrm{CW}=$ Chest width and $\mathrm{PW}=$ Pin bone width

Conclusions: All the body measurements have significant correlation coefficients while chest girth has the maximum value. Chest girth body measurement has also significant and consistent positive regression coefficient value to predict live body weight in Beetal goats in all age classes.

Acknowledgements: The study was supported by PAKUSAID funded research project "Collaborative Research for Genetic Conservation and Improvement of Pakistani Goats" at PMAS-Arid Agriculture University Rawalpindi, Pakistan and partially by EFS funded research project "Improvement of Beetal Goats and Indigenous Chicken through Dissemination of Superior Sires" at University of Agriculture Faisalabad, Pakistan that is highly acknowledged.

\section{REFERENCES}

Adeyinka, I.A. and I.D. Mohammed (2006). Relationship of Liveweight and Linear Body Measurement in
Two Breeds of Goat of Northern Nigeria. J. Anim. Vet. Adv. 5(11): 891-893.

An, X.P., J.G. Wang, J.X. Hou, H.B. Zhao, L. Bai, G. Li, L.X. Wang, X.Q. Liu, W.P. Xiao, Y. X. Song, and B. Y. Cao (2011). Polymorphism identification in the goat MSTN gene and association analysis with growth traits. Czech J. Anim. Sci. 56(12): 529-535.

Chitra, R., S. Rajendran, D. Prasanna, and A. Kirubakaran (2012). Prediction of body weight using appropriate regression model in adult female Malabari goat, Vet World, 5(7):409-411.

Eder, D., C. Aegerter, and K. Basler (2017). Forces controlling organ growth and size. Mechanisms of Development. 144(1):53-61.

Eisen, E. J. (1976). Results of Growth Curve Analyses in Mice and Rats1, 2. J. Anim. Sci.42:1008-1023.

Eyduran, E., M. Topal, A.Y. Sonmez, and S. Keskin (2012). Carcass weight estimation from some morphological traits of Capoeta capoeta capoeta (Güldenstädt, 1772) using factor scores in 
multiple regression analysis. Pakistan J. Stat. 28(1): 159-165

Eyduran, E., A. Waheed, M. Tariq, F. Iqbal, and S. Ahmad (2013). Prediction of live weight from morphological characteristics of commercial goat in Pakistan using factor and principal component scores in multiple linear regression. The J. Anim. Plant Sci. 23(6): 1532-1540.

Eyduran, E., D. Zaborski, A. Waheed, S. Celik, K. Karadas, and W. Grzesiak (2017) Comparison of the predictive capabilities of several data mining algorithms and multiple linear regression in the prediction of body weight by means of body measurements in the indigenous Beetal goat of Pakistan. Pakistan J. Zool. 49(1): 273-282.

Fahim, A., B. H. M. Patel, and V.V. Rijasnaz (2013). Relationship of body weight with linear body measurements in Rohilkhand local goats. Indian J. Anim. Res. 47(6):521-526.

GOP [Government of Pakistan], (2006). Pakistan Livestock Census. Agricultural Cencus Organization, Statistics Division, Lahore.

GOP [Government of Pakistan], (2018-19). Economic Survey of Pakistan 2018-19. [http://www.finance.gov.pk/survey/chapters_19/ 2-Agriculture.pdf].

Iqbal, M., K. Javed, and N. Ahmad (2013). Prediction of body weight through body measurements in Beetal goats. Pakistan J. Sci., 65(4):458-461.

Khan, M. A, M.M. Tariq, E. Eyduran, A. Tatliyer, M. Rafeeq, F. Abbas, N. Rashid, M. A. Awan, and K. Javed (2014). Estimating body weight from several body measurements in Harnai sheep without multicollinearity problem. The J. Anim. Plant Sci. 24(1):120-126.

Khan, H., F. Muhammad, R. Ahmad, G. Nawaz, Rahimullah, and M. Zubair (2006). Relationship of Body Weight with Linear Body Measurements in Goats. J. Agric. Biol. Sci. 1(3): 51-54.

Khan, M.S., M.A. Khan, and S. Mahmood (2008). Genetic resources and diversity in Pakistani goats. Int. J. Agric. Biol., 10:227-231.
Moela, A.K. (2014). Assessment of the relationship between body weight and body measurements in indigenous goats using Path analysis. M.Sc. Thesis. Deptt. of Agri. Econo. and Anim. Prod., Univ. Limpopo (South Africa).

Otoikhian, C., A. Otoikhian, O. Akporhuarho, V. Oyefia, and C. Isidahomen (2008). Body measurement parameters as a function of assessing body weight in goats under on-farm research environment. Afr. J. Gen. Agri. 4(3):135-140.

Pesmen, G., and M. Yardimci (2008). Estimating the live weight using some body measurements in Saanen goats. Archiva Zootechnica 11(4):30-40.

SAS, (2017). SAS University Edition. SAS Institute, Inc., Cary, NC.

Seifemichael, M., K. Kefelegn, A. Negassi, and A.K. Banerjee (2014). Variability in Linear Body Measurements and their Application in Predicting Body Weight of Afar Goats in Ethiopia. Int. J. Interdiscip. Multidiscip. Stud. 1(4):17-25.

Slippers, S.C., B.A. Letty, and J.F. De Villiers (2000). Prediction of the body weight of Nguni goats. S. Afr. J. Anim. Sci. 30(1):127-128.

Tariq, M.M., E. Eyduran, M.A. Bajwa, A. Waheed, F. Iqbal, and Y. Javed (2012). Prediction of body weight from testicular and morphological characteristics in indigenous Mengali sheep of Pakistan: using factor analysis scores in multiple linear regression analysis. Int. J. Agric. Biol., 14(4): 590-594.'

Tsegaye, D., B. Belay, and A. Haile (2013). Linear body measurements as predictor of body weight in hararghe highland goats under farmers environment: Ethiopia. Global Vet. 11(5):649656.

Waheed, A., (2011). Characterization of Goats for Linear Type Traits in Pakistan. PhD thesis. Deptt. of Anim. Breed. and Genet., Univ. Agri. Faisalabad. 